\title{
EVALUATION OF EYE CARE AND OCULAR FINDINGS IN CRITICALLY ILL COVID-19 PATIENTS
}

\author{
Zarife Ekici Gök ${ }^{1}$, Abdullah Gök ${ }^{1}$, leman acun delen² ${ }^{2}$ Umut Sabri Kasapoglu ${ }^{3}$, Esra \\ Gürbüz ${ }^{4}$, and Kayhan Mutlu ${ }^{5}$ \\ ${ }^{1}$ Malatya Training and Research Hospital \\ ${ }^{2}$ Malatya Egitim ve Arastirma Hastanesi \\ ${ }^{3}$ Malatya Turgut Özal University \\ ${ }^{4}$ Van Eğitim ve Araştırma Hastanesi \\ ${ }^{5}$ Aksaray University
}

September 25, 2021

\begin{abstract}
Objective: The current study aimed to evaluate eye care needs along with ocular findings in patients treated in intensive care units due to Covid-19 infection. Materials and Methods: A total of 93 patients, 58 men and 35 women, who were under follow-up and treatment in intensive care due to COVID-19 infection between 01/01/2021 and 02/15/2021 were included in the study. Detailed eye examinations of the patients were performed with a portable handheld biomicroscope and direct and indirect ophthalmoscope. Cases requiring treatment for eye diseases were identified and treated. Results: The mean patient age was $68.32 \pm 9.97$ years; 35 patients were followed-up and treated with non-invasive mechanical ventilation and 58 patients with invasive mechanical ventilation support. Mild, moderate, and severe chemosis was observed in 19 patients (20.4\%), 10 patients $(10.8 \%)$, and 4 patients $(4.3 \%)$, respectively. Conjunctivitis $(8.6 \%)$ was observed in 8 patients. Corneal abrasion was present in 7 patients $(7.5 \%)$. Keratitis secondary to exposure keratopathy was observed in one patient. Eye care and medical treatment was initiated for these patients. We noted 8 patients (8.6\%) with retinal haemorrhage; however, to the best of our knowledge, these patients may show acute retinal involvement secondary to systemic diseases or have previous retinal findings other than Covid-19 infection or treatments. Conclusion: Findings of ocular involvement due to COVID-19 infection were detected in the present study. Retinal haemorrhages were detected in addition to the common findings in viral infections. Especially in patients supported with mechanical ventilation, the detection and treatment of conditions that require eye care for exposure keratopathy is very important for recovering from the disease and the quality of vision.
\end{abstract}

\section{EVALUATION OF EYE CARE AND OCULAR FINDINGS IN CRITICALLY ILL COVID-19} PATIENTS

\section{ABSTRACT}

Objective: The current study aimed to evaluate eye care needs along with ocular findings in patients treated in intensive care units due to Covid-19 infection.

Materials and Methods: A total of 93 patients, 58 men and 35 women, who were under follow-up and treatment in intensive care due to COVID-19 infection between 01/01/2021 and 02/15/2021 were included in the study. Detailed eye examinations of the patients were performed with a portable handheld biomicroscope and direct and indirect ophthalmoscope. Cases requiring treatment for eye diseases were identified and treated. 
Results: The mean patient age was $68.32 \pm 9.97$ years; 35 patients were followed-up and treated with non-invasive mechanical ventilation and 58 patients with invasive mechanical ventilation support. Mild, moderate, and severe chemosis was observed in 19 patients (20.4\%), 10 patients (10.8\%), and 4 patients $(4.3 \%)$, respectively. Conjunctivitis $(8.6 \%)$ was observed in 8 patients. Corneal abrasion was present in 7 patients $(7.5 \%)$. Keratitis secondary to exposure keratopathy was observed in one patient. Eye care and medical treatment was initiated for these patients. We noted 8 patients $(8.6 \%)$ with retinal haemorrhage; however, to the best of our knowledge, these patients may show acute retinal involvement secondary to systemic diseases or have previous retinal findings other than Covid-19 infection or treatments.

Conclusion: Findings of ocular involvement due to COVID-19 infection were detected in the present study. Retinal haemorrhages were detected in addition to the common findings in viral infections. Especially in patients supported with mechanical ventilation, the detection and treatment of conditions that require eye care for exposure keratopathy is very important for recovering from the disease and the quality of vision.

Key words : Covid-19, eyecare, retina, ocular complications, critical care

\section{What's known}

The coronavirus has been shown to cause serious eye diseases in animals but ocular symptoms in humans are rare and mild. Recent studies have defined endothelial damage as one of the most prominent causes of systemic vascular thromboembolic and inflammatory manifestations associated with Covid-19. Sars-CoV-2 may exhibit direct ocular diffusion through two blood-retinal barriers (BRBs) or associated with thrombotic sensitivity identified in Covid-19.

\section{What's new}

Retinal haemorrhages and cotton wool spots were detected in addition to the common findings in viral infections. Eyecare in the intensive care unit and the application of treatments appropriate for ocular problems that may occur due to Covid-19 infection are vital for those who recover from Covid-19 infection to prevent vision loss and maintain quality of life.

\section{Introduction}

Covid-19, which has spread around the world leading to severe acute respiratory syndrome and was declared a pandemic by the World Health Organization (WHO), was detected in Turkey for the first time on 11 March 2020 (1).

Studies have shown that Sars-CoV-2 needs ACE-2 receptors infecting cells (2). ACE-2 receptors are also found in the cornea and conjunctiva besides the lung alveoli (3). This suggests that the ocular surface tissue may be the target tissue of the Sars-CoV-2 virus. Considering the similarities in coronavirus species, Covid-19 infection has been shown to cause ocular symptoms in patients (4).

Although ocular findings such as conjunctivitis, chemosis, epiphora, and conjunctival injection have been detected since the initial reports of Covid-19, it has also been reported that these findings may be the first sign of Covid -19 infection (5). In various case reports, retinal haemorrhage and retinal vein occlusion have been shown as complications of the posterior segment associated with Covid. In a published report, patients without any systemic disease and decreased vision after the onset of Covid -19 infection and found to have unilateral or bilateral retinal vein occlusion were reported. The report suggested that there may be a vein occlusion associated with vasculitis and thromboembolism due to immune complex accumulation $(6,7)$. In another report published, Paracentral Acute Middle Maculopathy and Acute Macular Neuroretinopathy were reported in a case of Covid -19 due to the hyper-reflective lesions detected in OCT as a result of decreased vision (8).

Since Covid-19 is a life-threatening infection, other organ interactions have not been fully determined, as many studies focus on the respiratory system to improve survival. Extra-pulmonary symptoms of Sars-Cov-2 should not be ignored, as they may be important causes of morbidity and a source of viral transmission. Therefore, the current study aimed to evaluate the ocular effects of Covid-19 infection and the treatments 
applied, especially in intensive care patients with severe disease, and to identify and treat patients who would require eye care.

\section{Material and Methods:}

In the current study, we included 93 patients who were being followed up and treated in the intensive care unit between $01 / 01 / 2021$ and $02 / 15 / 2021$ because of the complications associated with Covid-19 infection such as severe pneumonia, acute respiratory distress syndrome and multi-organ dysfunction. Both eyes of the patients were evaluated separately. Patient relatives were informed before the study and a signed consent form was obtained. The principles of the Declaration of Helsinki were complied with at all stages of the study. Ethical approval was obtained from the Clinical Research Ethics Committee (Decision number: 2021/21).

The diagnostic criterion for including patients in the study was a positive reverse transcription-PCR (RTPCR) test of the nasopharyngeal swab sample for SARS-CoV-2. 3 patients with a negative PCR test but typical Covid-19 appearance on chest CT scan and typical clinical symptoms, and patients of high clinical suspicion with high $\mathrm{C}$ reactive protein $(\mathrm{CRP})$ and lymphopenia $<1100 / \mathrm{mm} 3$ in the blood sample were also included in the study.

Demographic findings such as age and gender of the patients were determined, and the presence of systemic diseases was evaluated. Treatments applied for Covid-19 infection and laboratory parameters, especially coagulation factors, were evaluated. Personal protective equipment was worn and precautions were taken before entering the patient rooms. The anterior segment findings such as cornea, conjunctiva, and eyelids were examined first with a portable handheld biomicroscope and direct ophthalmoscope for each patient. Subsequently, the eyes were dilated with $1 \%$ Tropicamide, and retinal and optic nerve examinations were performed by the same ophthalmologist with an indirect ophthalmoscope and 28D lens. During the anterior segment examinations of the patients, conditions requiring eye care, especially in patients who were intubated and placed in the prone position, such as corneal epithelial defect, conjunctivitis and keratitis were determined. Intensive care personnel were also trained, and eye care and treatments were provided.

Statistical Analysis : The sample size of the study was determined by Power Test with the power of each variable considered at least $80 \%$ and type 1 error taken as $5 \%$. We checked whether the continuous measurements in the study were normally distributed or not with the Kolmogorov-Smirnov test $(\mathrm{n}>50)$ and Skewness-Kurtosis tests. Parametric tests were applied because the measurements were normally distributed. Descriptive statistics for continuous variables were expressed as mean, standard deviation, minimum, maximum. "Independent T-test" was used to compare the measurements between the groups. "Pearson correlation coefficients" were calculated to determine the relationships between measurements. Chi-square test was used to determine the relationship between categorical variables. In the calculations, the statistical significance level (a) was taken as 5\% and the SPSS (IBM SPSS for Windows, ver.24) statistics package program was used for analysis

\section{Results}

Mean patient age was $68.32 \pm 9.97$ years, 35 patients were followed up and treated with non-invasive mechanical ventilation and 58 patients with invasive mechanical ventilation support (Table 1). Patients were treated in the hospital for an average of 7 days from the onset of Covid-19 symptoms. Due to the clinical worsening, the patients were followed and treated in the intensive care unit, and ophthalmological examination was also performed in the intensive care unit. Ophthalmological examinations were performed after an average of 12.43 (2-30) days of admission to intensive care (Table 2). It was observed in the files of the patients that no ocular symptoms were reported in any of the patients.

Patients who developed severe chemosis were those who were occasionally placed in the prone position for treatment. Severe keratitis due to lagophthalmos was detected in 1 patient, conjunctivitis with the purulent discharge was detected in 1 patient, and eye closure and drug treatment (moxifloxacin and artificial tear without preservative) were initiated. When these patients were examined for control purposes, improvement 
was observed in patients with corneal abrasion, but treatment responses could not be followed up in the patient with keratitis and purulent conjunctivitis considering that the patients were dead. In two of the cases with conjunctivitis, PCR test was performed from conjunctival swabs to detect SARS-CoV-2, but a negative result was obtained.

Apart from retinal involvement due to systemic diseases, new intraretinal haemorrhages were observed in 8 patients $(8.6 \%)$. Bilateral haemorrhages were observed in 1 patient and splinter haemorrhages in the temporal side of the optic disc were observed in 2 patients. Cotton wool spots together with splinter haemorrhage in the temporal side of the optic disc were observed in 1 patient. In 4 patients, intraretinal haemorrhages were observed in the macula in the arch. One of the patients who had haemorrhage in the arch also had cerebral haemorrhage and bilateral papilloedema. There was no statistically significant difference in both comorbid systemic diseases and findings of laboratory parameters of these patients. The distribution and comparison of systemic disease and laboratory parameters of patients with retinal haemorrhage compared to other patients are shown in Table 3.

Of the 93 patients followed, 19 (20\%) patients survived, whereas other patients were lost due to complications due to Covid-19. 14 (15.05\%) of the 19 patients were followed up and treated with non-invasive ventilation, $5(5.3 \%)$ were treated with mechanical ventilation, and their treatment continued in the ward when the intensive care requirements decreased.

\section{Discussion}

Patients who are followed up and treated in the intensive care unit due to Covid-19 infection, in mechanical ventilation-including non-invasive ventilation-and at the prone position, are at risk for the development of ocular complications. The present study aimed to evaluate the eye care of patients recovering from Covid-19 infection to prevent vision loss as a result of ocular complications and to evaluate whether there is any ocular involvement during Covid-19 infection.

Although the main target of the SARS-CoV-2 virus is the respiratory tract, it is stated that it affects many other systems and that various symptoms are observed (9). Furthermore, coronavirus has been shown to cause serious eye diseases such as retinal vasculitis, anterior uveitis, optic neuritis in animals, ocular symptoms in humans are rare and mild (10).

The retina is considered as an extension of the central nervous system (CNS), therefore involvement may occur not only in the optic nerve (optic neuritis) but also in the retina (retinitis) in neuroinflammatory conditions and infections of the CNS (11). The CNS is protected from viruses by its multilayer barriers and the immune system, but viruses can affect the brain in various conditions such as direct brain damage, hypoxic damage, upregulated ACE-2 receptors, and immunodeficiency (12). It is well-known that ACE-2 receptors are widely distributed among many tissues and cell types, as well as in the conjunctiva. Some reports have shown that CoV-2 could potentially spread through direct or indirect contact with the mucous membranes of the eyes (13-15). Recent clinical and anatomopathological studies have defined endothelial damage as one of the most prominent causes of systemic vascular thromboembolic and inflammatory manifestations associated with Covid-19 (16-18). Therefore, vascular occlusion due to thrombotic sensitivity, chorioretinitis or vasculitis directly caused by the virus may occur in the retina. Considering that Sars-CoV-2 may exhibit direct ocular diffusion through two blood-retinal barriers (BRBs) or associated with thrombotic sensitivity identified in Covid-19, as reported in the brain, 8 of our patients who underwent retinal examination had intraretinal haemorrhages. Splintered haemorrhages in the temporal side of the optic disc were observed in 1 patient bilaterally and in 2 patients in the left eye. The Cotton wool spot was observed with a splinter haemorrhage in the temporal side of the optic disc in 1 patient. In 4 patients, intraretinal haemorrhages were observed in the macula in the arch. Marinho et al. detected microhemorrhages and cotton wool spots along the retinal arcade in 4 of 12 patients (19). But since cotton wool spots can be identified in a wide range of diseases $(20,21)$ and the comorbidities of patients that could lead to this condition were not specified, it was stated that it is impossible to definitively determine whether these were pathological cotton wool spot conditions (22). In the present study, one patient with a cotton wool spot and retinal haemorrhage in the temporal side 
of the optic disc had diabetes mellitus, but there were no findings of diabetic retinopathy other than this haemorrhage. No statistically significant difference was observed between patients with haemorrhage in the retina and other patients in terms of both systemic diseases and laboratory parameters.

In three different studies conducted in China, Xia et al. could not find any relationship between the severity of Covid-19 infection and the frequency of conjunctivitis (23), whereas Guan et al (24) and Wu et al. (15) showed that the incidence of conjunctivitis and other ocular symptoms were higher in patients with severe pneumonia. In the present study, conjunctivitis was seen with a rate of $8.6 \%$. The mechanism of how conjunctivitis occurs is still not fully understood. It may be endothelial dysfunction, vasculitis or the host response of conjunctival vessels due to Covid-19 infection (25).

More than $15 \%$ of patients affected by the Covid-19 pandemic are hospitalized and treated, a significant portion of which will require mechanical ventilation (26). Decrease in orbicular muscle tone due to neuromuscular blocking agents applied in patients under MV support and turning patients to the prone position, which is one of the treatment stages, increases the risk of exposure keratopathy in intensive care patients (27). Exposure keratopathy can be seen in patients who are connected to mechanical ventilation, especially in intensive care, and it can lead to progressive vision loss by causing microbial keratitis and ocular surface scarring (28)

A guideline has been approved by The Royal College of Ophthalmologists (RCOphth) in collaboration with the Intensive Care Association to prevent keratopathy in intensive care patients. It contains precautions that can be taken together with closing the eyelids (27). Kam et al. also suggested an eye care protocol in an eye care study in critically ill patients, emphasizing that clinicians should evaluate the closure of the eyelids in terms of lagophthalmos (29). To prevent the progression to keratopathy, eyelid closure and medical treatment was performed in patients in our intensive care unit with corneal epithelial defects seen in the first examinations during the examination phase. The intensive care staff caring for the patients was trained about the risk factors for developing keratopathy and the necessary preventive measures. Subsequently, the presence of corneal epithelial defect and keratopathy, which was observed in the first examinations with a rate of $7.5 \%$, was not observed in the subsequent examinations. Six patients requiring treatment and care due to conditions such as corneal epithelial defect, conjunctivitis and keratitis could not be followed up because of complications related to Covid-19. It was observed that the corneal epithelial defect in one patient resolved in two days after eye care.

The incidence of chemosis in the conjunctiva in critically ill patients ranges from $9 \%$ to $80 \%$ (30). Chemosis is classified as mild, moderate or severe according to the degree of conjunctival prolapse. Vascular permeability and lymph duct dysfunction in the conjunctiva lead to chemosis (31). Mechanical ventilation increases jugular venous pressure and causes fluid to accumulate in ocular tissues. Long-term positive pressure ventilation and fluid electrolyte abnormalities in intensive care are factors that increase conjunctival chemosis (32). Chemosis may cause keratopathy and related complications by causing irregular tear distribution on the ocular surface according to its severity (33). Against the risk of developing keratopathy due to severe chemosis, especially in patients who are placed in the prone position, ocular evaluation should be performed before the patients are placed in the prone position, and eyelids should be taped by applying pomade to the eye (34). Our patients were hospitalized for a long time due to Covid-19 infection, and mild chemosis was observed at a rate of $20.4 \%$, moderate chemosis at a rate of $10.8 \%$, and severe chemosis at a rate of $4.3 \%$, especially in patients who were intubated. It was observed that lagophthalmos developed due to oedema in the eyelids in one patient, and it was observed that severe chemosis and exposure keratopathy developed. Eyelid closure and lubrication treatment were initiated for the patient, and considering that closing the eyelids would not be sufficient, tarsoraphy was planned in the patient but treatment and follow-up could not be performed because the patient died due to complications related to Covid-19 infection the next day.

Schwartz et al. stated that subconjunctival bleeding may be more frequent in patients with Covid-19 infection treated in intensive care (35). In another study, subconjunctival haemorrhage was observed at a rate of $8.3 \%$ (36). In the present study, subconjunctival bleeding was observed in $3(3.2 \%)$ patients. One of these cases was in the form of punctate bleeding foci with chemosis. It is thought that these bleedings may due to severe 
cough, vomiting due to intolerance of the drugs used, or due to the effect of anticoagulant drugs given in treatment to eliminate the risk of hypercoagulability of Covid-19 infection.

In the present study, it was aimed to prevent ocular complications that may occur in all critical patients in the intensive care unit by providing eye care information such as daily examination of the eyes, lubrication in the presence of lagophthalmos and closing the eyelids by taping. Eyecare in the intensive care unit and the application of treatments appropriate for ocular problems that may occur due to Covid-19 infection are vital for those who recover from Covid-19 infection to prevent vision loss and maintain quality of life.

The present study was conducted on patients who were being treated in intensive care. For this reason, as a limitation of our study, it was not possible to distinguish whether retinal haemorrhages were due to disease or drug use. We think that the application of systemic treatments to prevent the complications of Covid-19 infection before ophthalmological examination and hypoxia due to the disease makes it difficult to distinguish whether these findings are caused by Covid-19 infection or the treatment.

As a result, corneal abrasion due to lagophthalmos was observed in $10.3 \%$ of the patients who were followed up in the intensive care unit. Care should be taken in terms of exposure keratopathy, both due to not treating corneal abrasions, and also secondary to the development of severe chemosis and eyelid oedema in patients who are placed in the prone position. Daily eye examinations are required, especially in critical cases of Covid-19 under mechanical ventilation support.

We observed retinal haemorrhage at a rate of $8.6 \%$. We observed that Covid-19 infection can directly cause endothelitis, thromboembolism or retinal haemorrhage and cotton wool spots due to the treatments used. There was no significant difference in terms of systemic disease and laboratory parameters in patients with haemorrhage in the retina compared to those without haemorrhage.

\section{Declaration of competing interest}

The authors have no conflicts of interest to disclose.

\section{Acknowledgement}

None declared.

\section{Author Contributions}

ZEG, AG, LAD, USK, KM and EG contributed to the design and conceptualization of the study. ZEG, AG, LAD, USK contributed to indesigning the search strategy and literatüre search. Data extraction was conducted by ZEG an AG. Data entry and statistical analysis were performed EG. Critical appraisal was done by ZEG, USK and KM. ZEG and KM contributed to drafting and writing the manuscript. All authors reviewed and contributed in editing the manuscript.

\section{References}

1. Cucinotta D, Vanelli M. WHO declares COVID-19 a pandemic. Acta Biomed. 2020;91:157-160. viro3. Seah I, Agrawal R. Can the coronavirus disease 2019 (COVID-19) affect the eyes? A review of coronaviruses and ocular implications in humans and animals. Ocul Immunol Inflamm. 2020;28:391-395.

2. Wan Y, Shang J, Graham R, et al. Receptor recognition by the novel coronavirus from Wuhan: an analysis based on decade-long structural studies of SARS coronavirus. J Virol 2020; 94(7): e00127-20.

3. Zou X, Chen K, Zou J, et al. Single-cell RNA-seq data analysis on the receptor ACE2 expression reveals the potential risk of different human organs vulnerable to 2019-nCoV infection. Front Med 2020; 14(2): $185-192$.

4. Yuen KS, Chan W-M, Fan DS, et al. Ocular screening in severe acute respiratory syndrome. Am J Ophthalmol. 2004;137 (4):773-774. doi:10.1016/S0002-9394(03)01148-6. 
5. Seah IYJ, Anderson DE, Kang AEZ, et al. Assessing viral shedding and infectivity of tears in Coronavirus Disease 2019 (COVID-19) Patients [published online ahead of print, 2020 Mar 24]. Ophthalmology. 2020; S0161-6420(20)30311-0.

6. Walinjkar, J. A., Makhija, S. C., Sharma, H. R., Morekar, S. R., \& Natarajan, S. (2020). Central retinal vein occlusion with COVID-19 infection as the presumptive etiology. Indian Journal of Ophthalmology, 68(11), 2572.

7. Gaba, W. H., Ahmed, D., Al Nuaimi, R. K., Al Dhahani, A. A., \& Eatmadi, H. (2020). Bilateral Central Retinal Vein Occlusion in a 40-Year-Old Man with Severe Coronavirus Disease 2019 (COVID-19) Pneumonia. The American Journal of Case Reports, 21, e927691-1.

8. Virgo, J., \& Mohamed, M. (2020). Paracentral acute middle maculopathy and acute macular neuroretinopathy following SARS-CoV-2 infection. Eye, 1-2

9. Wang C, Horby PW, Hayden FG, Gao GF. A novel coronavirus outbreak of global health concern. Lancet. 2020;395:470-473.

10. Seah I, Agrawal R. Can the coronavirus disease 2019 (COVID-19) affect the eyes? A review of coronaviruses and ocular implications in humans and animals. Ocul Immunol Inflamm. 2020;28:391-395

11. Zhou Y, Zeng Y, Tong Y, Chen C. Ophthalmologic evidence against the interpersonal transmission of 2019 novel coronavirus through conjunctiva. MedRxiv. 2020.

12. Wu Y, Xu X, Chen Z, Duan J, Hashimoto K, Yang L et al (2020) Nervous system involvement after infection with COVID-19 and other coronaviruses. Brain, Behav Immunity. 87:18-22

13. Jun ISY, Anderson DE, Kang AEZ, Wang L-F, Rao P, Young BE et al (2020) Assessing viral shedding and infectivity of tears in coronavirus disease 2019 (COVID-19) patients. Ophthalmology 127(7):977-979

14. Sun X, Zhang X, Chen X, Chen L, Deng C, Zou X, et al. The infection evidence of SARS-COV-2 in ocular surface: a single-center cross-sectional study. MedRxiv. 2020.

15. Wu P, Duan F, Luo C, Liu Q, Qu X, Liang L et al (2020) Characteristics of ocular findings of patients with coronavirus disease 2019 (COVID-19) in Hubei Province. China, JAMA ophthalmology

16. Bikdeli, B. et al. COVID-19 and Thrombotic or Thromboembolic Disease: Implications for Prevention, Antithrombotic Therapy, and Follow-up. Journal of the American College of Cardiology (2020). doi:10.1016/j.jacc.2020.04.031

17. England, J. T. et al. Weathering the COVID-19 storm: Lessons from hematologic cytokine syndromes. Blood Reviews (2020). doi:10.1016/j.blre.2020.100707

18. Copin, M. C. et al. Time to consider histologic pattern of lung injury to treat critically ill patients with COVID-19 infection. Intensive Care Medicine (2020). doi:10.1007/s00134-020-06057-8

19. Marinho, P. M., Marcos, A. A. A., Romano, A. C., Nascimento, H. \& Belfort, R. Retinal ndings in patients with COVID-19. The Lancet 395, 1610 (2020)

20. Brown GC, Brown MM, Hiller T, Fischer D, Benson WE, Magargal LE. Cotton-wool spots. Retina. $1985 ; 5: 206-14$.

21. Ioannides A, Georgakarakos ND, Elaroud I, Andreou P. Isolated cotton-wool spots of unknown etiology: management and sequential spectral domain optical coherence tomography documentation. Clin Ophthalmol. 2011;5:1431-3.

22. Vavvas, D.G. et al. Concerns about the interpretation of OCT and fundus findings in COVID-19 patients in recent Lancet publication. Eye 1-2 (2020). doi:10.1038/s41433-020-1084-9 
23. Xia J, Tong J, Liu M, Shen Y, Guo D. Evaluation of coronavirus in tears and conjunctival secretions of patients with SARS-CoV-2 infection. J Med Virol. 2020;92:589-594.

24. Guan WJ, Ni ZY, Hu Y, et al. Clinical characteristics of coronavirus disease 2019 in China. N Engl J Med. 2020;382:1708-1720.

25. Oncul, Hasan, et al. "Ocular findings in patients with coronavirus disease 2019 (COVID-19) in an outbreak hospital." Journal of medical virology 93.2 (2021): 1126-1132.

26. Kolifarhood G, Aghaali M, Saadati HM, et al. Epidemiological and clinical aspects of COVID-19; a narrative review. Archives of Academic Emergency Medicine 2020; 8: e41

27. Hearne BJ, Hearne EG, Montgomery H, Lightman SL. Eyecare in the intensive care unit. Journal of the Intensive Care Society 2018; 19: 345-50.

28. Kousha O, Kousha Z, Paddle J. Exposure keratopathy: Incidence, risk factors and impact of protocolised care on exposure keratopathy in critically ill adults. Journal of Critical Care 2018; 44: 413-8.

29. Kam KR, Haldar S, Papamichael E, Pearce KC, Hayes M, Joshi N: Eyecare in the critically ill: a national survey and protocol. J Intensive Care Soc. 2013, 14:150-154. 10.1177/175114371301400213

30. Mercieca F, Suresh P, Morton A, Tull, A: Ocular surface disease in intensive care unit patients. Eye. 1999, 2:231-236. 10.1038/eye.1999.57

31. Pacella SJ, Codner MA: Minor complications after blepharoplasty: dry eyes, chemosis, granulomas, ptosis, and scleral show. Plast Reconstr Surg. 2010, 125:709-718. 10.1097/PRS.0b013e3181c830c7

32. Imanaka H, Taenaka N, Nakamura J, Aoyama K, Hosotani, H: Ocular surface disorders in the critically ill. Anesth Analg. 1997, 85:343-346. 10.1097/00000539-199708000-00018

33. Grixti A, Sadri M, Watts MT. Corneal protection during general anaesthesia for nonocular surgery. Ocular Surface. 2013;11(2):109-118.

34. Intensive Care Society and Faculty of Intensive Care Medicine. Guidance for: prone positioning in adult critical care. 2019. https://www.ficm.ac.uk/sites/default/files/prone_position_in_adult_critical_care_2019.pdf (accessed 18 May 2020)

35. Schwarz L, Lwowski C, Schmack I, et al. Ophthalmological care of SARS-CoV-2 positive patients in intensive care units. Ophthalmologe. 2020;117:602-608.

36. Guemes-Villahoz N, Burgos-Blasco B, Arribi-Vilela A, et al. Detecting SARS-CoV-2 RNA in conjunctival secretions: is it a valuable diagnostic method of COVID-19? [published online ahead of print June 24, 2020]. J Med Virol. 2020.

\section{Hosted file}

Tables.docx available at https://authorea.com/users/436477/articles/538767-evaluation-ofeye-care-and-ocular-findings-in-critically-ill-covid-19-patients 